\title{
American College of Medical Genetics guideline on the cytogenetic evaluation of the individual with developmental delay or mental retardation
}

Lisa G. Shaffer, PhD, on behalf of the American College of Medical Genetics (ACMG) Professional Practice and Guidelines Committee

Key Words: mental retardation, developmental delay, cytogenetic analysis, karyotype, chromosomes

Disclaimer: This guideline is designed primarily as an educational resource for medical geneticists and other health care providers to help them provide quality medical genetic services. Adherence to this guideline does not necessarily assure a successful medical outcome. This guideline should not be considered inclusive of all proper procedures and tests or exclusive of other procedures and tests that are reasonably directed to obtaining the same results. In determining the propriety of any specific procedure or test, the geneticist should apply his or her own professional judgment to the specific clinical circumstances presented by the individual patient or specimen. It may be prudent, however, to document in the patient's record the rationale for any significant deviation from this guideline.

The following are the recommendations of the American College of Medical Genetics (ACMG) Professional Practice and Guidelines Committee, which was convened to assist health care professionals in making decisions regarding cytogenetic diagnostic testing and counseling for mental retardation (MR) and developmental delay (DD). This document reviews available evidence concerning the value of conventional and molecular cytogenetic testing for the identification of chromosomal anomalies that play a role in the etiology of MR/ $\mathrm{DD}$, and, based on this evidence, specific recommendations for each method of testing are provided.

\section{INTRODUCTION}

Mental retardation (MR), defined by the World Health Organization (WHO) as an intelligence quotient (IQ) $<70$, is characterized by significantly limited cognitive functioning, coupled with limitations in adaptive skills in two or more of the following areas: social skills, community living, communication, home living, health, self-direction, work, and leisure. ${ }^{1,2}$ MR is generally divided into mild (IQ of 50-70), moderate (IQ of 35-50), and severe (IQ of 20-35); those cases in which the IQ is below 20 are occasionally defined as profound..$^{2,3}$ The prevalence rate of MR in the general population is estimated to be approximately $1 \%$ to $3 \%$, with mild MR occurring 7-10 times more frequently than moderate or severe MR.2,4,5

\footnotetext{
From the Health Research and Education Center, Washington State University Spokane, WA. American College of Medical Genetics, 9650 Rockville Pike, Bethesda, MD 20814-3998. Approved by the Board of Directors October 26, 2004.

Go to www.geneticsinmedicine.org for a printable copy of this document. DOI: 10.1097/01.gim.0000186545.83160.1e
}

Global developmental delay (DD) describes significant delay in two or more of the following areas: cognition, speech/ language, gross/fine motor skills, social/personal skills, and daily living. ${ }^{6} \mathrm{DD}$ is evidenced as age-specific deficits in learning skills and adaptation in comparison with chronological peers. ${ }^{6}$ Because the diagnosis of MR requires accurate and valid assessments of intelligence, the term DD is generally reserved for children five years of age or younger, prior to the age at which IQ testing can be applicable. Thus, a child with DD is not necessarily destined to have MR. Several conditions, such as cerebral palsy, some neuromuscular disorders, or adverse environmental effects, may result in early childhood learning delay, though the child may test in the normal range for intelligence when he or she is old enough to be accurately assessed. Like $\mathrm{MR}$, the prevalence rate of DD in the general population is estimated to be 1 to $3 \% .^{6-10}$

The etiology of MR/DD is complex and may include environmental factors, Mendelian disorders, and chromosomal abnormalities, presenting alone or in combination. The cause of MR/DD can be identified in $40 \%$ to $60 \%$ of cases; ${ }^{1}$ however, the percentage of mild MR/DD cases for which a cause can be established is $24 \%$, which is significantly lower. ${ }^{11}$ Chromosome abnormalities are the single most common cause found in series of unselected patients with MR/DD. Rates of identifying chromosome abnormalities range from $15 \%$ to $40 \%$ in surveys of severe MR/ DD, with substantially lower rates for mild MR/DD. ${ }^{11,12}$ Furthermore, it is estimated that approximately $5 \%$ of MR/DD can be attributed to chromosomal abnormalities in the subtelomeric regions. ${ }^{13,14}$ In addition, individuals with learning disabilities and/or speech delays, with or without behavior problems, although not mentally retarded, are also more likely to have chromosome abnormalities, particularly 47,XXX, 47,XXY, or 47,XYY. Chromosome abnormalities are also found in approximately $5 \%$ 
of individuals with autism, usually in association with MR/DD. ${ }^{15}$ Thus, cytogenetic studies can reveal the cause in a substantial proportion of cases of MR/DD.

An accurate diagnosis for MR/DD is valuable for the patient and the patient's family as well as for health care providers. An early diagnosis can elucidate the recurrence risk for parents, especially those carrying chromosome rearrangements. Therapeutic strategies can be investigated more easily, appropriate counseling and intervention issues can be addressed, and important diagnostic and treatment information can be provided to schools and care providers. ${ }^{1}$

A set of guidelines for diagnosis and genetic testing would be useful, given the variety and complexity of causes of MR/DD, and the benefits of an accurate diagnosis. The following are the recommendations of the American College of Medical Genetics (ACMG) Professional Practice and Guidelines Committee, which was convened to assist health care professionals in making decisions regarding cytogenetic diagnostic testing and counseling for MR/DD. This document reviews available evidence concerning the value of conventional and molecular cytogenetic testing for the identification of chromosomal anomalies that play a role in the etiology of MR/DD, and, based on this evidence, specific recommendations for each method of testing are provided.

\section{RECOMMENDATIONS FOR TESTING}

\section{Routine chromosome analysis}

Historically, when investigating an individual with MR or DD, with or without dysmorphic features, the initial analysis began with routine chromosome analysis of peripheral blood. Developed in the 1970s, G-banding and other chromosome banding techniques permit the identification of the alternating light and dark staining bands comprising each chromosome, the detection of aneuploidy (extra or missing chromosomes), and the identification of microscopically apparent structural aberrations, including deletions and translocations. The routine G-banding level of blood chromosome analysis is in the range of 400-550 bands per haploid karyotype; however, for patients with MR or developmental delay, constitutional studies with banding levels of $<550$ should be repeated (ACMG Standards and Guidelines for Clinical Genetics Laboratories, Section E5.1.1.4). Banding techniques are routine procedures in clinical cytogenetic laboratories and are important in the evaluation of all individuals with MR or developmental delay regardless of the presence of dysmorphic features, small stature, congenital abnormalities, or unusual or manneristic behavior. It should not be assumed that perinatal distress and delivery complications are adequate to explain subsequent developmental delay and MR, because these complications can be associated with chromosome abnormalities. Based on good and consistent evidence, the overall yield of routine cytogenetic testing is $3.7 \%{ }^{6}$

\section{High-resolution chromosome analysis}

Because the chromosomal alterations that result in MR/DD frequently are undetectable at the level of resolution of routine chromosomal analysis, high-resolution analysis may be required to detect microdeletions, microduplications, or subtle translocations in the individual with MR/DD. Rather than using cells in mid-metaphase, which display bands that have fused during contraction, high-resolution chromosomal analysis involves the synchronization of lymphocyte cultures to achieve a population of cells in prophase or prometaphase. ${ }^{16}$ Whereas the metaphase cells used in conventional chromosomal analysis yield approximately 400-550 bands per haploid genome, prophase or prometaphase preparations enable the visualization of up to 1000 bands. ${ }^{17}$ Because of the increased number of visible bands, small structural chromosomal abnormalities such as deletions or duplications not resolvable with standard karyotypes may be detected. The ACMG Standards and Guidelines for Clinical Genetics Laboratories recommends that a focused high-resolution analysis should be reserved for cases in which a specific microabnormality syndrome is being considered, the diagnosis of which generally requires chromosomes above the 650-band stage (resolution at the 850-band level may be necessary). In addition, complete high-resolution chromosome analysis should always include a minimum of three pairs of each chromosome at a level of resolution above the 650-band stage. However, because of the increased amount of work required and the difficulty of high-resolution chromosomal analysis, routinely achieving approximately 850 bands is usually reserved for targeting specific chromosomal regions. ${ }^{18}$ At resolutions $>650$ bands, alterations as small as 3-5 Mb can be reliably detected using chromosome analysis on peripheral blood; for the detection of subtle rearrangements in patients with either abnormal or normal karyotypes, molecular cytogenetic analysis may be useful.

\section{Molecular cytogenetic analysis}

For individuals with MR/DD and phenotypes suggestive of particular disorders, targeted fluorescence in situ hybridization (FISH) can be performed prior to or concurrent with routine chromosome analysis if a particular microdeletion/microduplication syndrome is under consideration. FISH involves the hybridization of fluorescently labeled DNA probes to the denatured DNA of metaphase chromosomes or interphase nuclei directly on a glass slide. ${ }^{19-21}$ Types of DNA probes include unique sequence probes such as:

- bacterial artificial chromosome (BAC) clones, yeast artificial chromosome (YAC) clones, and cosmid clones that are used to interrogate a specific locus on a chromosome;

- repetitive sequence probes, such as alpha-satellite sequences to identify the centromeric regions of individual chromosomes; or

- whole-chromosome painting (WCP) probes, which utilize chromosomal libraries of clones for hybridization along the length of a specific chromosome.

FISH is capable of detecting submicroscopic $(<5 \mathrm{Mb})$ alterations that cannot be reliably observed using routine or high-resolution microscopic chromosome analysis. Resolution by FISH is dictated by the size of the probes used. For 
example, single cosmids provide a resolution of approximately 30 to $40 \mathrm{~kb}$, while contigs of overlapping cosmids or BAC probes provide a resolution of 80 to $200 \mathrm{~kb} .^{18}$ The importance of FISH is illustrated by its use in detecting submicroscopic rearrangements in numerous microdeletion syndromes, including those with a relatively high frequency in the general population (e.g., DiGeorge/velocardiofacial syndrome, approximately 1:400022), and some microduplication syndromes (e.g., dup (17)(p11.2p11.2) ${ }^{23}$ ). However, because FISH relies upon the use of locationspecific probes, it can only be used when the phenotype suggests a particular disorder so a specific probe can be requested. If locus-specific FISH analyses reveal abnormalities, FISH should also be performed on both parents to identify a carrier parent. FISH screening on patients with moderate to severe MR/DD has been shown to have a relatively high yield of $6.8 \% .^{6}$

\section{Subtelomeric analysis}

Because idiopathic MR/DD accounts for a significant proportion of MR/DD cases-approximately $40 \%$ of moderate to severe $\mathrm{MR} / \mathrm{DD}^{11}$ and $45 \%$ to $65 \%$ of mild $\mathrm{MR} / \mathrm{DD}^{24}$-and because most of these individuals do not have a specific phenotype that would indicate the use of a locus-specific probe, locus-specific FISH is not feasible for most initial attempts at diagnosis. However, evidence exists that aberrations in the terminal bands of chromosomes, the subtelomeric regions, are the cause of many cases of idiopathic MR/DD. Although reports of subtelomeric aberrations in unexplained MR/DD vary by their inclusion criteria, number and type of subjects, and testing acumen, it is estimated that approximately $5 \%$ of unexplained cases of MR/DD can be attributed to alterations of the subtelomeric regions. ${ }^{13,14,25}$ This is because the subtelomeric regions are relatively gene-rich, ${ }^{26}$ with alterations in these regions predicted to be more likely to result in abnormal phenotypes, and are believed to be prone to rearrangement due to a number of mechanisms. ${ }^{27,28}$ Because the ends of chromosomes lack distinctive G-bands, it is difficult to see small rearrangements at these regions in routine karyotype analysis and sometimes even at higher resolution (850-band). Furthermore, the presence of repetitive sequences within the telomeric regions precludes the use of chromosome-specific telomeric probes for FISH analysis due to the potential for multiple hybridization signals. However, the development of a set of 41 unique subtelomere probes (the short arms of the acrocentric chromosomes are not represented, and the $\mathrm{X} / \mathrm{Yp}$ and $\mathrm{X} / \mathrm{Yq}$ pseudoautosomal regions are represented by one clone each $)^{29,30}$ has permitted analysis of the unique regions approximately $300 \mathrm{~kb}$ proximal to the repetitive telomere region.

The degree of developmental delay/mental retardation or learning disability is a major predictor of the likelihood of finding a subtelomeric rearrangement as the cause of the individual's disorder. Those with moderate or severe MR/DD and a small chromosomal abnormality are more likely to have some combination of facial dysmorphism, minor physical abnormalities of the hands or feet, small stature, and/or microcephaly. The finding of MR/DD and an abnormal phenotype in a relative increases the chances of detecting a small chromosomal rearrangement. ${ }^{31}$ Recently, a set of clinical criteria for subtelomere screening was proposed $^{32}$ that may be helpful in some cases. The authors concluded that good indicators for subtelomeric defects are positive family history, prenatal-onset growth retardation, postnatal poor growth/overgrowth, two or more facial dysmorphic features, and one or more nonfacial dysmorphic features and/or congenital abnormalities. ${ }^{32}$ If subtelomere FISH analyses reveal abnormalities, FISH should also be performed on both parents to establish the parental origin of the anomaly and exclude the possibility of polymorphism..$^{33,34}$

\section{Subsequent testing after finding a structural chromosome abnormality}

After initial testing, if the patient's chromosomes display a structural abnormality, chromosome analysis should be performed on both parents to determine whether one of them carries a balanced rearrangement. If a carrier parent is detected, the nature of the rearrangement should become clear. If parental chromosomes are normal, several molecular cytogenetic techniques, used alone or in tandem (e.g., FISH using WCP probes or subtelomere probes) can be used to define the patient's rearrangement.

In addition, techniques have been developed to differentially label each human chromosome with a unique color combination to obtain 24-color karyotypes. ${ }^{35-38}$ This allows the detection of structural chromosomal aberrations at a level of resolution similar to that of a routine karyotype ( 400 to 550 bands). However, these techniques cannot detect rearrangements involving only a single chromosome such as deletions, inversions, and intrachromosomal duplications, and the limited number of spectrally resolvable fluorochromes may prohibit accurate detection of some translocations. ${ }^{39}$ Thus, 24-color karyotyping is usually used in conjunction with other procedures (e.g., subtelomere FISH) to maximize effectiveness.

\section{Subsequent testing options if no chromosome abnormality is found}

Direct DNA analysis for the fragile $\mathrm{X}$ syndrome is indicated in addition to chromosome analysis for all developmentally delayed/mentally retarded individuals, both male and female, who do not have an apparent underlying cause for their clinical status such as microcephaly, craniofacial dysmorphism and multiple congenital abnormalities. ${ }^{40}$ Both chromosome analysis and fragile X DNA analysis should be performed because chromosome abnormalities are as common, or more common, than fragile $\mathrm{X}$ mutations in mentally retarded individuals. ACMG recommendations for diagnostic testing for fragile $\mathrm{X}$ syndrome, a significant inherited cause of MR/DD, have been published previously. ${ }^{40}$ The overall yield of abnormal results after fragile $\mathrm{X}$ testing is $\sim 2.6 \%{ }^{6}$

If, after initial testing by karyotyping and molecular cytogenetics the patient's chromosome analysis reveals no abnormal- 
ity, and fragile X DNA analysis is negative, several options remain. Comparative genomic hybridization (CGH) is one possible option. In this technique, two genomes are differentially labeled and directly compared by simultaneous hybridization to normal metaphase chromosomes. It has an average resolution of approximately $10 \mathrm{Mb}$ in most laboratories, ${ }^{41-43}$ although higher resolutions have been reported. ${ }^{44}$ However, for most laboratories the low resolution precludes its usefulness. The future application of CGH is on microarrays (array $\mathrm{CGH}$ ), in which the two differentially labeled genomes are compared using large-insert clones immobilized on a glass slide as the substrates for analysis. ${ }^{45,46}$ With this technique the resolution is substantially increased over conventional cytogenetics, depending on the size and density of clones used. It is likely that for certain applications, such as the individual with nonspecific MR/DD, array CGH may be used in conjunction with routine cytogenetics to replace the locus-specific and/or subtelomere FISH in the future. ${ }^{47}$

\section{Analysis of tissues other than blood}

The routine and molecular cytogenetic analyses outlined thus far are usually performed on peripheral blood. However, there are some situations in which the cytogenetic analysis of a skin biopsy or other tissue may be indicated. First is the analysis of a different tissue type in T-cell deficient individuals. Because the analysis of metaphase chromosomes requires stimulation of T-cells by a mitogen (phytohemagglutinin) to enter mitosis, some individuals, such as those with DiGeorge syndrome, may not have enough normal T-cells to yield adequate metaphases for analysis. In these cases, skin fibroblast cultures, which do not depend on phytohemagglutinin stimulation, can be used to evaluate the chromosomes.

A second example is the cytogenetic analysis of skin fibroblasts to detect mosaicism. Current standard methods for cytogenetic analysis of peripheral blood will identify only those chromosome aberrations that are present in the circulating T-lymphocytes. However, there are certain chromosome abnormalities that do not appear to be compatible with survival of the T-cells. ${ }^{48}$ Therefore, only those T-cells that do not contain the aberration will be present in the circulating lymphocytes. Examples of this include mosaic tetrasomy of the short arm of chromosome 12 (Pallister-Killian syndrome ${ }^{49,50}$ ) and mosaic trisomy $17 .{ }^{51}$

Finally, the chromosomal aberration simply may not be present in the peripheral blood because of tissue-limited mosaicism. ${ }^{51}$ In cases in which chromosomal analysis is normal but suspicion of a chromosome abnormality remains high, a skin fibroblast culture can be used to detect the aberration. Skin biopsies may be taken from individuals with asymmetry or individuals with hypo- and hyperpigmented regions. The skin should be cleansed with alcohol and not with iodine-containing compounds because these inhibit cell growth in culture. Chromosome analysis of skin biopsies has been used to study parents who have more than one child with the same chromosome aberration, e.g., trisomy $21 .^{52}$ Case reports suggest that cytogenetic analysis of skin biopsies is a possible alter- native when chromosome analysis of blood is normal, although the yield is unknown. Studies using series of patients are needed to determine the overall yield for finding a cytogenetic diagnosis using skin biopsies.

\section{Analysis when fetal karyotype is normal following prenatal diagnosis}

In cases of developmental delay or MR, with or without physical anomalies, and in newborns with dysmorphic features and/or multiple malformations, a blood karyotype should be performed even when a fetal karyotype from amniocentesis or chorionic villus sampling is normal. There are several reasons to perform a blood karyotype. First, there may be mosaicism that has gone undetected in the fetal analysis. Second, the banding resolution is often higher in postnatal blood preparations than in cultured amniocytes or chorionic villi. Finally, the majority of fetal chromosome analyses are done for general reasons (e.g., advanced maternal age, abnormal triple-marker screening) and not for a specific set of dysmorphic features. After birth, the clinical features may provide clues regarding a specific chromosomal imbalance or for the use of additional techniques (e.g., FISH).

\section{Summary of Recommendations}

- For any child with unexplained MR/DD, even in the absence of dysmorphic facial features, other clinical features or positive family history, routine chromosome analysis (minimum 550-band resolution) is indicated.

- For children with clinical features of known chromosomal abnormality syndromes (e.g., Down syndrome), cytogenetic analysis should be performed. The identification of a translocation may affect the family's recurrence risk.

- High-resolution chromosome analysis is not routinely indicated unless a specific chromosomal region is to be investigated or there is a family history of a particular abnormality. These studies should be limited in focus and used when FISH is not available.

- For children with clinical features suggestive of a particular microdeletion/microduplication syndrome, FISH or other molecular techniques should be performed prior to or concurrently with chromosome analysis.

- If chromosome analysis is normal at 550-band resolution, subtelomere FISH testing may be considered.

\section{ACKNOWLEDGMENTS}

The authors thank Maureen Bocian, M.D. (University of California Irvine Medical Center, Orange, CA), Lawrence Shapiro, M.D. (New York Medical College and Westchester Medical Center, Valhalla, NY), Stuart Schwartz, Ph.D. (University of Chicago, Chicago, IL) and Kurt Hirschhorn, M.D. (Mount Sinai School of Medicine, New York, NY) for their contributions to the manuscript; and Daynna Wolff, Ph.D. (Medical University of South Carolina, Columbia, SC), Christa Lese Martin, Ph.D. (Emory University, Atlanta, GA), David Ledbet- 
ter, Ph.D. (Emory University, Atlanta, GA), Jon Zonana, M.D. (Oregon Health Sciences University, Portland, OR), Debra Driscoll, M.D. (University of Pennsylvania Medical Center, Philadelphia, PA) for their critical reviews of the manuscript; and Aaron Theisen (Washington State University, Spokane, WA) for his editorial support.

\section{References}

1. Curry CJ, Stevenson RE, Aughton D, Byrne J, et al. Evaluation of mental retardation: Recommendations of a Consensus Conference: American College of Medical Genetics. Am J Med Genet 1997;72:468-477.

2. Battaglia A, Bianchini E, Carey JC. Diagnostic yield of the comprehensive assessment of developmental delay/mental retardation in an institute of child neuropsychiatry. Am J Med Genet 1999;82:60-66.

3. Chiurazzi P, Oostra BA. Genetics of mental retardation. Curr Opin Pediatr 2000;12: 529-535.

4. Bundey S, Thake A, Todd J. The recurrence risks for mild idiopathic mental retardation. J Med Genet 1989;26:260-266.

5. Lamont MA, Dennis NR. Aetiology of mild mental retardation. Arch Dis Child 1988;63:1032-1038.

6. Shevell M, Ashwal S, Donley D, Flint J, et al. Practice parameter: Evaluation of the child with global developmental delay. Report of the Quality Standards Subcommittee of the American Academy of Neurology and The Practice Committee of the Child Neurology Society. Neurology 2003;60:367-380.

7. Yeargin-Allsopp M, Murphy CC, Cordero JF, Decoufle P, et al. Reported biomedical causes and associated medical conditions for mental retardation among 10-year-old children, metropolitan Atlanta, 1985 to 1987. Dev Med Child Neurol 1997;39:142-149.

8. Batshaw ML, Shapiro BK. Mental retardation. In: Batshaw ML, editor. Children with disabilities, 4th ed. Baltimore: Paul H. Brookes, 1997:335-359.

9. Kinsbourne M, Graf WD. Disorders of mental development. In: Menkes JH, Sarnat HB, editors. Child neurology, 6th ed. Philadelphia: Lippincott Williams \& Wilkins, 2001:1155-1211.

10. Simeonsson RJ, Simeonsson NW. Development surveillance and intervention. In: Hoekelman RA, Adam HM, Nelson NM, Weitzman ML, Wilson MH, editors. Primary pediatric care, 4th ed. St. Louis: Mosby, 2001:274-282.

11. Flint J, Wilkie AO. The genetics of mental retardation. Br Med Bull 1996;52:453-464.

12. Raymond GV. Abnormal mental development. In: Rimoin DL, Connor M, Pyeritz RE, Korf BR, editors. Emery and Rimoin's principles and practice of medical genetics. London: Churchill-Livingstone, 2002:1046-1065.

13. Flint J, Wilkie AO, Buckle VJ, Winter RM, et al. The detection of subtelomeric chromosomal rearrangements in idiopathic mental retardation. Nat Genet 1995;9: 132-140.

14. Knight SJ, Regan R, Nicod A, Horsley SW, et al. Subtle chromosomal rearrangements in children with unexplained mental retardation. Lancet 1999;354:1676-1681.

15. Lauritsen M, Mors O, Mortensen PB, Ewald H. Infantile autism and associated autosomal chromosome abnormalities: A register-based study and a literature survey. J Child Psychol Psychiatry 1999;40:335-345.

16. Yunis JJ. High resolution of human chromosomes. Science 1976;191:1268-1270.

17. Yunis JJ. Mid-prophase human chromosomes. The attainment of 2000 bands. Hum Genet 1981;56:293-298.

18. Shaffer LG, Ledbetter DH, Lupski JR. Molecular cytogenetics of contiguous gene syndromes: mechanisms and consequences of gene dosage imbalance. In: Scriver CR, Beau$\operatorname{det}$ AL, Sly WS, Valle D, Childs B, Kinzler KW, Vogelstein B, editors. The metabolic and molecular basis of inherited disease. New York: McGraw Hill, 2001:1291-1324.

19. Jauch A, Daumer C, Lichter P, Murken J, et al. Chromosomal in situ suppression hybridization of human gonosomes and autosomes and its use in clinical cytogenetics. Hum Genet 1990;85:145-150.

20. Lichter P, Boyle AL, Cremer T, Ward DC. Analysis of genes and chromosomes by nonisotopic in situ hybridization. Genet Anal Tech Appl 1991;8:24-35.

21. Trask BJ. Fluorescence in situ hybridization: Applications in cytogenetics and gene mapping. Trends Genet 1991;7:149-154.

22. Devriendt K, Fryns JP, Mortier G, van Thienen, MN, et al. The annual incidence of DiGeorge/velocardiofacial syndrome. J Med Genet 1998;35:789-790.

23. Potocki L, Chen KS, Park SS, Osterholm DE, et al. Molecular mechanism for duplication 17p11.2- the homologous recombination reciprocal of the Smith-Magenis microdeletion. Nat Genet 2000;24:84-87.

24. McLaren J, Bryson SE. Review of recent epidemiological studies of mental retardation: Prevalence, associated disorders, and etiology. Am J Ment Retard 1987;92:243-254.
25. Biesecker LG. The end of the beginning of chromosome ends. Am J Med Genet 2002;107:263-266.

26. Saccone S, De Sario, A, Della Valle, G, Bernardi G. The highest gene concentration in the human genome are in telomeric bands of metaphase chromosomes. Proc Natl Acad Sci U S A 1992;89:4913-4917.

27. Ballif BC, Wakui K, Gajecka M, Shaffer LG. Translocation breakpoint mapping and sequence analysis in three monosomy 1 p36 subjects with der $(1) t(1 ; 1)(p 36 ; q 44)$ suggest mechanisms for telomere capture in stabilizing de novo terminal rearrangements. Hum Genet 2004;114:198-206.

28. Ballif BC, Yu W, Shaw CA, Kashork CD, et al. Monosomy 1 p36 breakpoint junctions suggest pre-meiotic breakage-fusion-bridge cycles are involved in generating terminal deletions. Hum Mol Genet 2003;12:2153-2165.

29. A complete set of human telomeric probes and their clinical application. Nationa Institutes of Health and Institute of Molecular Medicine collaboration. Nat Gene 1996;14:86-89.

30. Knight SJ, Horsley SW, Regan R, Lawrie NM, et al. Development and clinical application of an innovative fluorescence in situ hybridization technique which detects submicroscopic rearrangements involving telomeres. Eur J Hum Genet 1997;5:1-8.

31. Knight SJ, Flint J. Perfect endings: A review of subtelomeric probes and their use in clinical diagnosis. J Med Genet 2000;37:401-409.

32. de Vries BB, White SM, Knight SJ, Regan R, et al. Clinical studies on submicroscopic subtelomeric rearrangements: a checklist. J Med Genet 2001;38:145-150.

33. Ballif BC, Kashork CD, Shaffer LG. The promise and pitfalls of telomere regionspecific probes. Am J Hum Genet 2000;67:1356-1359.

34. Shaffer LG, Kashork CD, Bacino CA, Benke PJ. Caution: Telomere crossing. Am J Med Genet 1999;87:278-280.

35. Schrock E, du Manoir, S, Veldman T, Schoell B, et al. Multicolor spectral karyotyping of human chromosomes. Science 1996;273:494-497.

36. Uhrig S, Schuffenhauer S, Fauth C, Wirtz A, et al. Multiplex-FISH for pre- and postnatal diagnostic applications. Am J Hum Genet 1999;65:448-462.

37. Schrock E, Veldman T, Padilla-Nash H, Ning Y, et al. Spectral karyotyping refines cytogenetic diagnostics of constitutional chromosomal abnormalities. Hum Genet 1997;101:255-262.

38. Speicher MR, Gwyn Ballard, S, Ward DC. Karyotyping human chromosomes by combinatorial multi-fluor FISH. Nat Genet 1996;12:368-375.

39. Fauth C, Speicher MR. Classifying by colors: FISH-based genome analysis. Cytogenet Cell Genet 2001;93:1-10.

40. Sherman S, Pletcher BA, Driscoll DA. Fragile X syndrome: Diagnostic and carrier testing. American College of Medical Genetics Professional Practice and Guidelines Committee. Genet Med 2005;7:584-587.

41. Bentz M, Plesch A, Stilgenbauer S, Dohner H, et al. Minimal sizes of deletion detected by comparative genomic hybridization. Genes Chromosomes Cancer 1998; 21:172-175.

42. Kallioniemi A, Kallioniemi OP, Sudar D, Rutovitz D, et al. Comparative genomic hybridization for molecular cytogenetic analysis of solid tumors. Science 1992;258: $818-821$.

43. Levy B, Dunn TM, Kaffe S, Kardon N, et al. Clinical applications of comparative genomic hybridization. Genet Med 1998;1:4-12

44. Kirchhoff M, Gerdes T, Maahr J, Rose H, et al. Deletions below 10 megabasepairs are detected in comparative genomic hybridization by standard reference intervals. Genes Chromosomes Cancer 1999;25:410-413.

45. Pinkel D, Segraves R, Sudar D, Clark S, et al. High resolution analysis of DNA copy number variation using comparative genomic hybridization to microarrays. Nat Genet 1998;20:207-211.

46. Solinas-Toldo S, Lampel S, Stilgenbauer S, Nickolenko J, et al. Matrix-based comparative genomic hybridization: biochips to screen for genomic imbalances. Genes Chromosomes Cancer 1997;20:399-407.

47. Shaffer LG, Bejjani BA. A cytogeneticist's perspective on genomic microarrays. Hum Reprod Update 2004;10:221-226.

48. Kulharya AS, Lovell CM, Flannery DB. Unusual mosaic karyotype resulting from adjacent 1 segregation of $\mathrm{t}(11 ; 22)$ : Importance of performing skin fibroblast karyotype in patients with unexplained multiple congenital anomalies. Am J Med Genet 2002;113:367-370

49. Hunter AG, Clifford B, Cox DM. The characteristic physiognomy and tissue specific karyotype distribution in the Pallister-Killian syndrome. Clin Genet 1985;28:47-53.

50. Peltomaki P, Knuutila S, Ritvanen A, Kaitila I, et al. Pallister-Killian syndrome: Cytogenetic and molecular studies. Clin Genet 1987;31:399-405.

51. Shaffer LG, McCaskill C, Hersh JH, Greenberg F, et al. A clinical and molecular study of mosaicism for trisomy 17. Hum Genet 1996;97:69-72.

52. Hsu LY, Gertner M, Leiter E, Hirschhorn K. Paternal trisomy 21 mosaicism and Down's syndrome. Am J Hum Genet 1971;23:592-601. 